\title{
Neue Melanomdiagnostik-Verfahren mit Vorsicht zu gebrauchen
}

\section{Zwei in jüngster Zeit in die dermatologische} Melanomdiagnostik eingeführte Verfahren sind in einer US-amerikanischen Studie miteinander verglichen worden. Völlig überzeugen konnte keines der beiden.

D ermatologen und Versorgungsforscher aus den USA untersuchten die Spezifität und Sensitivität einerseits der digitalen multispektralen Analyse von Hautläsionen (MDSLA) und andererseits der reflektierenden konfokalen Mikroskopie (RCM). Beide Techniken können bei suspekten pigmentierten Hautmalen als ergänzende diagnostische Maßnahmen herangezogen werden und sollen helfen, die Zahl unnötiger Exzisionen zu senken.

Die MDSLA (MelaFind) bedient sich zehn verschiedener Wellenlängen zwischen 430 und $950 \mathrm{~nm}$, um Informationen über die Eigenschaften des Hautmals bis zu einer Eindringtiefe von $2,5 \mathrm{~mm} \mathrm{zu}$ gewinnen. Daraus wird ein Score errechnet, der angibt, wie hoch die Wahrscheinlichkeit eines Melanoms ist. Für die RCM (VivaScope 1500) wird hingegen ein $830-\mathrm{nm}$-Diodenlaser verwendet. Sie nutzt die Reflektionseigenschaften der Hautstrukturen, um die Zellen von der Epidermis bis zur papillären Dermis mit einer Eindringtiefe von $0,2 \mathrm{~mm}$ sichtbar zu machen.

55 klinisch und dermatoskopisch atypische, melanomverdächtige Pigmentmale von 36 Patienten wurden in die Studie einbezogen. Sie wurden mit den genannten optischen Verfahren abgeklärt, anschlieBend biopsiert (Punch, Shave) oder exzidiert und histopathologisch begutachtet. Der histopathologische Befund diente als Goldstandard. In vier Fällen lag ein Melanoma in situ, in drei Fällen ein dysplastischer Nävus mit schweren Atypien und der Empfehlung einer erneuten Exzision vor. Damit ergab sich im untersuchten Kollektiv eine Rate exzisionspflichtiger Male von rund $13 \%$.

Zusätzliche diagnostische Verfahren wie MDSLA und RCM sollten in erster Linie kein Melanom übersehen und daher eine Sensitivität von nahe $100 \%$ erreichen. Das gelang in der vorliegenden Studie noch eher mit der RCM, deren Sensitivität $85,7 \%$ betrug. Die MDSLA kam auf $71,4 \%$. Die Spezifitätswerte lagen bei $66,7 \%$ und $25 \%$. Auch wenn die Spezifität in diesem Zusammenhang weniger wichtig sein mag, würden solch niedrige Werte - falls sie sich bestätigen - den prädiktiven Wert positiver RCM- und MDSLA-Resultate schmälern. Denn gemessen an der Prävalenz exzisionspflichtiger Läsionen ist die Rate falsch positiver Ergebnisse relativ hoch. Außerdem wäre dann zu bezweifeln, ob der Einsatz der Verfahren die Zahl unnötiger Exzisionen erheblich vermindern kann.

Fazit: Der Vergleich zwischen RCM und MDSLA in der Diagnostik suspekter pigmentierter Läsionen der Haut zeigt Vorteile für die RCM. Völlig überzeugen können aber beide Methoden nicht. Jedoch war die Zahl der analysierten Fälle relativ klein, und vor allem die MDSLA hatte in früheren Studien deutlich besser abgeschnitten. Robert Bublak

Song E et al. Paired comparison of the sensitivity and specificity of multispectral digital skin lesion analysis and reflectance confocal microscopy in the detection of melanoma in vivo: A cross-sectional study. J Am Acad Dermatol. 2016;75:118792.e2

\section{Hier steht eine Anzeige.}

\author{
照 Springer
}

